\title{
Analysis of Electron and Antineutrino Energy Spectra from Fis- sile Samples under Irradiation based on Gross Theory of Beta- decay
}

\author{
T. Yoshida ${ }^{1, a}$, T. Tachibana ${ }^{2}$, and S. Chiba ${ }^{1}$ \\ ${ }^{1}$ Tokyo Institute of Technology, Ookayama, Meguro-ku, Tokyo \\ ${ }^{2}$ Senior High School of Waseda University, Kamishakujii, Nerima-ku, Tokyo
}

\begin{abstract}
We applied the gross theory of $\beta$-decay to calculate the reactor electron and antineutrino $\left(\bar{v}_{e}\right)$ spectra emitted from ${ }^{235,238} \mathrm{U}$ and ${ }^{239,241} \mathrm{Pu}$ by summing up all the contributions from a large number of decaying fission-products (FPs). We make it clear what kinds of transition types and FP nuclides are important to shape the lepton spectra. After taking the ambiguity in the current data for fission yields and $Q_{\beta}$-values into account, we suggested a possibility that the high-energy part of the widely referred electron-spectra by Schreckenbach et al., almost only one experimental data set available now, might possibly be too low. Arguments on a special role of the $\operatorname{odd}(Z)-\operatorname{odd}(N)$ nuclides and on the consistency between U-238 and other fissiles in the experimental data lead to the importance of a new and independent measurement of electron energy spectra which could be converted into the reactor $\bar{v}_{e}$ spectra.
\end{abstract}

\section{Introduction}

Recently increasing attention is paid to the technology of antineutrino-based monitoring of nuclearreactor operation from the outside for non-proliferation purposes. Precise determination of the antineutrino energy-spectra is one of the key issues there. Reliable knowledge of the reactor antineutrino spectra is also required for interpreting the neutrino oscillation experiments for basic research of physics. One way to achieve this is to convert the measured electron spectra from fissionable samples into the antineutrino spectra using the energy conservation of leptons in the $\beta$-decay process. Another is to compose the spectrum by summing up all the contributions from a large number of decaying fission products (FPs).

\section{Calculation Method}

In order to describe the aggregate behaviour of FPs in a reactor core, we start with the calculation of the number of each FP nuclide considering its creation and destruction. By summing up the contributions from them, we can calculate the electron and the antineutrino $\left(\bar{v}_{e}\right)$ spectra as

$$
I_{e}\left(E_{e}\right)=\sum N_{i} \lambda_{i} I_{i}^{e}\left(E_{e}\right)
$$

\footnotetext{
ae-mail: tyoshida@nr.titech.ac.jp
} 


$$
I_{\bar{v}}\left(E_{\bar{v}}\right)=\sum N_{i} \lambda_{i} I_{i}^{\bar{v}}\left(E_{\bar{v}}\right)
$$

where $N_{i}$ and $\lambda_{i}$ are the number density and the decay constant of the $i$-th FP. The point is how to obtain the spectra, $I_{i}^{e}\left(E_{e}\right)$ and $I_{i}^{\bar{v}}\left(E_{\bar{v}}\right)$, characteristic of each nuclide $i$. Here $E_{e}$ and $E_{\bar{v}}$ are the kinetic energies of the electron and the antineutrino. In order to calculate $I_{i}^{e}\left(E_{e}\right)$ and $I_{i}^{\bar{\gamma}}\left(E_{\bar{v}}\right)$, we applied the Gross Theory of beta decay first developed by Takahashi, Koyama and Yamada[1-3] and then improved by Kondoh and Tachibana et al.[4, 5]. The details of these calculations related to eqs.(1) and (2) are given elsewhere[6]. The decay constants $\lambda_{i}$ and the decay chains were taken from JENDL FP Decay Data File 2011 along with the fission yields from JENDL Fission Yield Data File 2011 [7]. Hereafter we call these two files together as JENDL simply.

\section{Calculation Results}

The calculated results for electron spectra are shown in Fig. 1. The experimental data cited here are from a series of extensive measurements by the group of Schreckenbach et al. [8-11]. They measured the energy spectra of electron from fissionable-nuclide samples under irradiation using a spectrometer called BILL [12] at the Institute of Laue-Langevin. Hereafter we call this the 'BILL experiment.' The time-lapse from the beginning of irradiation, in accordance to which the number density $N_{i}$ in eqs.(1) and (2) were calculated here, is indicated in parentheses. In addition to this, another set of experimental data is available [13]. Though this measurement is rather old and covers only the energy range up to $8.5 \mathrm{MeV}$ for ${ }^{235} \mathrm{U}$, it is also an elaborative work (we call this the 'UI experiment' after University of Illinois).

As is seen in Figs. 2 and 3, the main contributor to the antineutrino spectra is the Gamow-Teller transition. This is followed by the first-forbidden transition which covers $15 \%$ (around 2-3 MeV) $20 \%$ (above $6 \mathrm{MeV}$ ) of the emitted antineutrinos. These two types overwhelm the Fermi-transition. Needless to say, this transition-wise break down of the antineutrino spectra is almost the same as the case of the corresponding electron spectra, which are not shown in Fig.1 so as to save the space. In these figures, three sets of $\bar{v}_{e}$ spectra converted from the measured electron spectra not only by measured themselves but by other people by their own sophisticated method.

\section{Discussions}

\subsection{Below $8 \mathrm{MeV}$}

Table 1 shows the $\mathrm{C} / \mathrm{E}$ values for the electron spectra at typical energy points. Though the agreement between the calculation and the measurement is not always satisfactory for the practical applications previously mentioned, it should be underlined that the overall trends of the spectra below $\sim 8 \mathrm{MeV}$ are reproduced fairly well in the absolute-value basis without any tuning of the parameters in the theory. There seems to be two noticeable points in Table 1. One is the fact the calculations are persistently larger than the measured values over the whole energy range. This suggests that the overestimation might be related to the calculation of the number of beta-decay events rather than to the individual spectrum shape of each FP nuclide. This exact point stimulates us to investigate further, where we treat the FP decay-heat data including the $\beta$-ray spectra at very short cooling times on the same basis as the reactor lepton-spectra dealt with here. Another point is that the overall trend of the $\mathrm{C} / \mathrm{E}$ for ${ }^{238} \mathrm{U}$ is different from others; that is, the $\mathrm{C} / \mathrm{E}(\sim 1.1)$ is closer to unity and less dependent on the electron energy. Considering that there had been a 25 -years lapse of time after the last ${ }^{241} \mathrm{Pu}$ measurements and that the neutron source had been changed from the ILL-reactor to the FRM-II facility, a possibility cannot be excluded that some unknown bias exists between the new ${ }^{238} \mathrm{U}$ and the old ${ }^{235} \mathrm{U}$ and $\mathrm{Pu}$ measurements, or otherwise in both. At least we cannot exclude this possibility one-hundred percent. 


\subsection{Above $8 \mathrm{MeV}$}

In all the cases from ${ }^{235} \mathrm{U}$ to ${ }^{241} \mathrm{Pu}$, the same kind of the conspicuous overestimation against the BILL data is seen above $\sim 8 \mathrm{MeV}$. It should be noted, however, the antineutrino with energy higher than 8 $\mathrm{MeV}$ has little importance as far as the current detection technique using the reaction $\bar{v}_{e}+p \rightarrow n+\bar{e}$ is premised. It is another puzzle that the UI curve, which is fairly consistent with the BILL-curve up to $7 \mathrm{MeV}$, suddenly deviates upward from BILL. It is noteworthy that even the contribution solely from the nuclides with $Q_{\beta}=10 \sim 12 \mathrm{MeV}$ exceeds the converted the $\bar{v}_{e}$ spectra above $8 \mathrm{MeV}$ as is seen in Fig.4. Here it must be recognised that the gross theory is an average theory which describes the average property of decaying nuclides specified by their mass-number and even/odd-ness. Therefore, the prediction power of this theory for an aggregate behaviour of a small number of FPs is inherently limited and it must be applied very carefully to the reactor lepton spectrums above $8 \mathrm{MeV}$, namely, our concerns here.

Table 2 lists the top 10 contributors which constitute the high energy part of the $\bar{v}_{e}$ spectra at 9 $\mathrm{MeV}$. It should be noted that the 8 odd-odd nuclides in Table 2 cover $78 \%$ of the spectral value at 9 $\mathrm{MeV}$. As the origin of the large discrepancy in the $\bar{v}_{e}$ spectra above $8 \mathrm{MeV}$, we first think about the fission yield data. The effect of whole replacement of the fission yields for the summation calculation from JENDL to JEFF-3.1[14] seems to be rather restricted as is seen in Fig.5. When we see in details, however, a big differences between JENDL (or ENDF-B/VII[15]) and JEFF3.1 can not be ignored. Figure 6 shows the mass yield for $A=88$ to which important contributers Br- 88 and As- 88 in Table 2 belong. The charge-yield curves in JENDL and ENDF/B-VII.1 has an unexpected shoulder on the lower- $Z$ side of the peak in contrast to the smooth behaviour seen in the JEFF-3.1. This is seen also in mass-chains $\mathrm{A}=86,96,100,102$, and 131 among others. This different behaviour gives rise to the spectrum variation between JENDL and JEFF as we saw in Fig.5 as a whole. Anyway, the uncertainty in the fission yields does not seem to be the origin of the big discrepancy above $8 \mathrm{MeV}$. In addition,as is argued in ref.[6], neither the ambiguity in $Q_{\beta}$ does explain the discrepancy.

\subsection{Selection Rules for Odd-Odd Nuclide Decay}

Here we have to remember fact that the prediction power of the gross theory for an aggregate behaviour of a small number of FPs is less reliable than large number cases because individual properties of few nuclides can play a decisive role beyond the realm of this average theory. From Table 2 we see that a limited number of odd $(Z)$-odd $(N)$ nuclides dominate the highest-energy part of the $\bar{v}_{e}$ spectra where transitions to the ground-state, if possible, might play the most important role.

Any $\beta$-decay of an odd-odd nuclide end up in an even-even nuclide whose low-lying levels are sparse compared to odd- $A$ and odd-odd nuclides, and this enhances the importance of its groundstate transition. As the ground-state of any even-even nuclide is determined to be $0^{+}$, we can easily estimate the effect of the selection rules on the $\beta$-transition to the low-lying states provided we know the spin-parity of the decaying odd-odd nuclide (see the last column of Table 2 where the latest NDS evaluation is shown). This kind of consideration was successfully applied to the reactor decay heat problem[17], and is expected to mitigate the serious disagreement above $8 \mathrm{MeV}$ in the electron and the $\bar{v}_{e}$ spectra.

\section{Concluding Remarks}

We applied the gross theory of $\beta$-decay to calculate the reactor electron and antineutrino $\left(\bar{v}_{e}\right)$ spectra emitted from ${ }^{235,238} \mathrm{U}$ and ${ }^{239,241} \mathrm{Pu}$ along with the summation methods. Though the agreement between the calculation and the measurement is not always satisfactory for the practical applications in 
nuclear technology and basic particle physics, it should be underlined that the overall trends of the spectra below $\sim 8 \mathrm{MeV}$ are reproduced fairly well in the absolute-value basis without any tuning of the parameters in the theory, which leaves promising room for further improvement. One aspect is a possible modification of the treatment of the spin-parity effect of decaying odd-odd nuclides. It would hopefully remedy the overestimation against the BILL-experimental data seen above $8 \mathrm{MeV}$ both in the $\bar{v}_{e}$ and the electron spectra. Throughout the present arguments, however, we could not exclude a possibility that the lepton spectra by Schreckenbach et al. (practically the only one extensive series of measurements) are not free from any systematic bias. Generally speaking it might be risky to rely on a single experimental data set in practical applications where very high precision is required, even if their assumed experimental errors are small.

\section{References}

[1] K. Takahashi, M. Yamada, Progr. Theor. Phys. 41, 1470-1503 (1969).

[2] S. I. Koyama, K. Takahashi, M. Yamada, Progr. Theor. Phys. 44, 663-687 (1970).

[3] K. Takahashi, Progr. Theor. Phys. 45, 1466-1492 (1971).

[4] T. Kondoh, T. Tachibana, M. Yamada, Progr. Theor. Phys. 74, 708-724 (1985).

[5] T. Tachibana, M. Yamada, Y. Yoshida, Progr. Theor. Phys. 84, 641-657 (1990),

[6] T. Yoshida, T. Tachibana, N. Hagura, S. Chiba, Progr. Nucl. Energy, 88, 175-182 (2016).

[7] J. Katakura, JENDL FP Decay Data File 2011 and Fission Yield Data File 2011, Japan Atomic Energy Agency, Report JAEA-Data/Code 2011-25 (2011).

[8] K. Schreckenbach, G. Colvin, W. Gelletly, F. Von Feilitzsch, Phys. Lett. 160B, 325-330 (1985).

[9] F. Von Feilitzsch, A. A. Hahn, K. Schreckenbach, Phys. Lett. 118B, 162-166 (1982).

[10] A. A. Hahn, K. Schreckenbach, W. Gelletly, F. Von Feilitzsch, G. Colvin, B. Krusche, Phys. Lett. B218, 365-368 (1989).

[11] N. Haag, A. Gütlein, L. Hofmann, L. Oberauer, W. Potzel, K. Schreckenbach, Phys. Rev. Lett. 112, 122501-1-5 (2014).

[12] W. Mampe, K. Schreckenbach, P. Jeuch, B. P. K. Maier, F. Braumandl, J. Larysz, T. von Egidy, Nucl. Instr. Meth. 154, 127-149 (1978).

[13] N. Tsoulfanidis, B. W. Wehring, M. E. Wyman, Nucl. Sci. Eng. 43, 42-53 (1971).

[14] M. A. Kelett, O. Bersillon, R. W. Mills, The JEFF-3.1/-3.1.1 radioactive decay data and fission data, OECD NEA Report N.6287, Nuclear Energy Agency/OECD (2009).

[15] M. B. Chadwick, et al., Nucl. Data Sheets 112, 2887-2996 (2011).

[16] M. Wang, G. Audi, A. H. Wapstra, F. G. Kondev, M. MacCormick, X. Xu, B. Pfeifer, Chinese Phys. C 36, 1603-2014 (2012).

[17] T. Yoshida, Nucl. Sci. Engn. 63, 376-390 (1977). 
Table 1. C/E (calculated/experimental) values of the electron spectra from ${ }^{235,238} \mathrm{U}$ and ${ }^{239,241} \mathrm{Pu}$ samples at typical energy points

\begin{tabular}{|c|c|c|c|c|}
\hline Energy (MeV) & U-235 & U-238 & Pu-239 & Pu-241 \\
\hline 2.0 & 1.18 & - & 1.25 & 1.28 \\
3.0 & 1.22 & 1.11 & 1.32 & 1.29 \\
4.0 & 1.15 & 1.11 & 1.29 & 1.24 \\
5.0 & 1.08 & 1.07 & 1.24 & 1.20 \\
6.0 & 1.01 & 1.11 & 1.25 & 1.26 \\
7.0 & 1.12 & - & 1.29 & 1.46 \\
8.0 & 3.75 & - & 2.38 & 2.91 \\
9.0 & 16.2 & - & - & 10.5 \\
\hline
\end{tabular}

Table 2. Top 10 nuclides contributing to $\mathrm{U} 235 \bar{v}_{e}$ spectrum at $9 \mathrm{MeV}$

\begin{tabular}{|c|c|c|c|}
\hline Nuclide & Q (MeV) & Fractional Contribution & Spin-Parity \\
\hline Rb-94 & 10.29 & 0.240 & $3^{-}$(Abriola,2006) \\
Y-98m & 9.23 & 0.121 & 4 or 5 (Singh,2003) \\
As-86 & 11.54 & 0.087 & not given (Negret, 2015) \\
Br-90 & 10.35 & 0.077 & not given (Browne, 1997) \\
Y-100m & 9.46 & 0.075 & 3,4 or 5 (Singh, 2008) \\
Br-88 & 8.96 & 0.068 & $2^{-}$(McCutchan,2014) \\
Rb-95 & 9.28 & 0.065 & - \\
As-84 & 10.09 & 0.042 & $3^{-}$(Abriola,2009) \\
As-88 & 12.60 & 0.038 & not given (McCutchan,2014) \\
Br-91 & 9.80 & 0.035 & - \\
\hline F.R.Sum & - & 0.848 & - \\
\hline
\end{tabular}




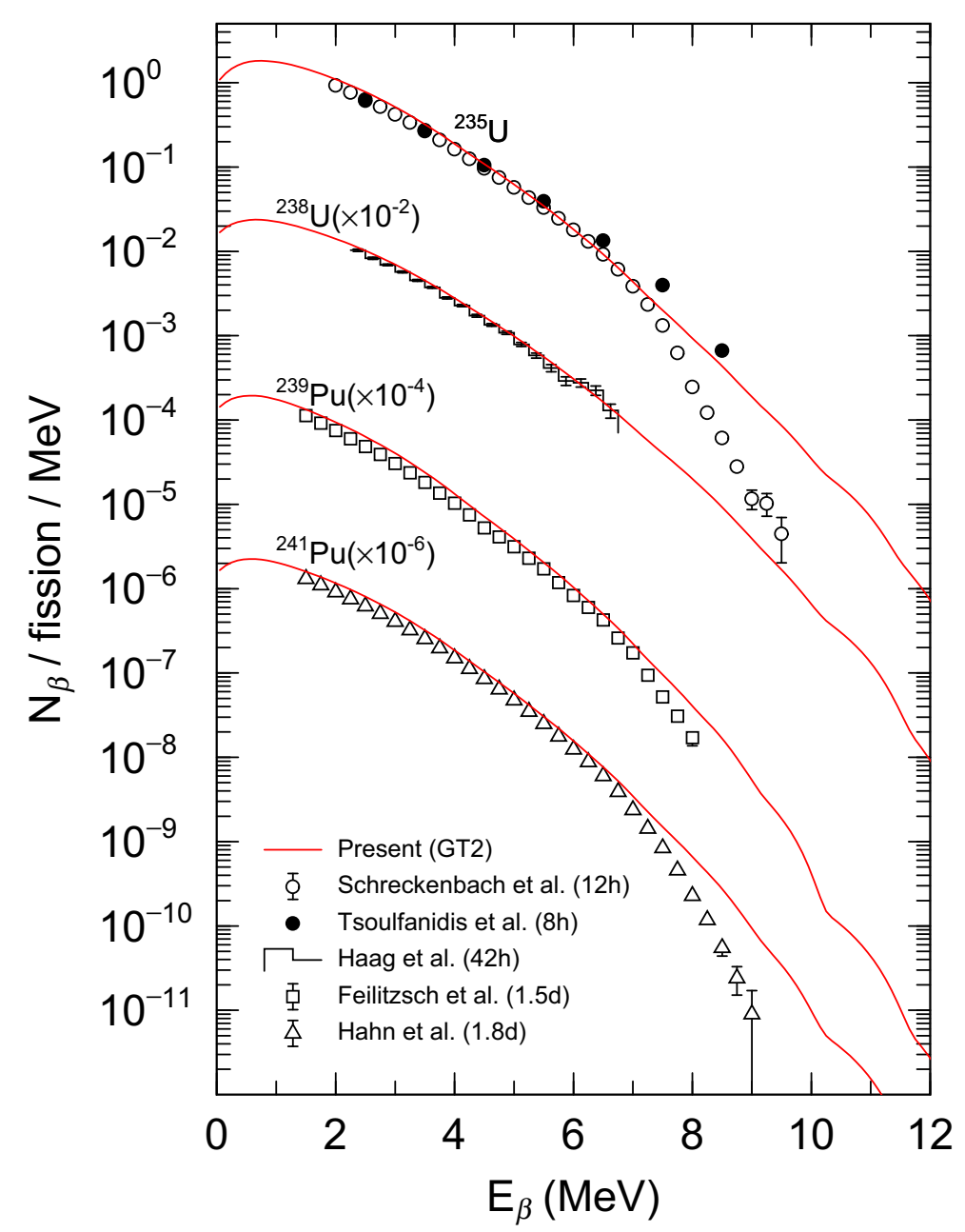

Figure 1. Comparison of the aggregate electron spectra from ${ }^{235,238} \mathrm{U}$ and ${ }^{239,241} \mathrm{Pu}$ samples between the present calculation and the measurements 


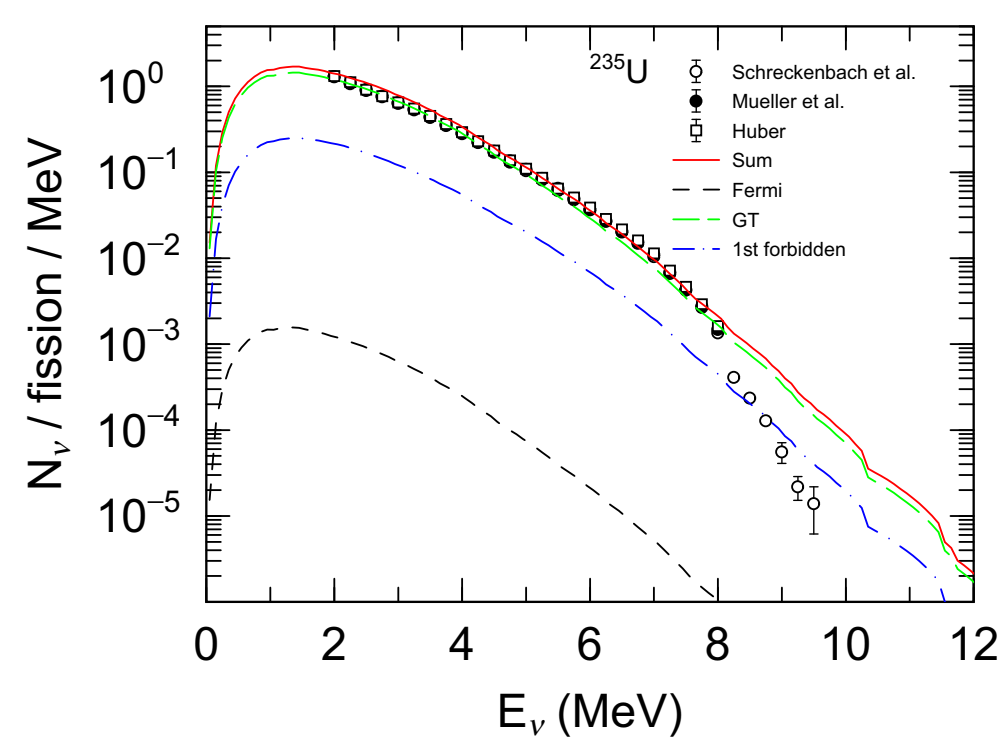

Figure 2. Calculated antineutrino spectrum from ${ }^{235} \mathrm{U}$ sample and its component-wise breakdown. For reference are shown three spectra converted from the electron spectrum measured by Schreckenbach et al.

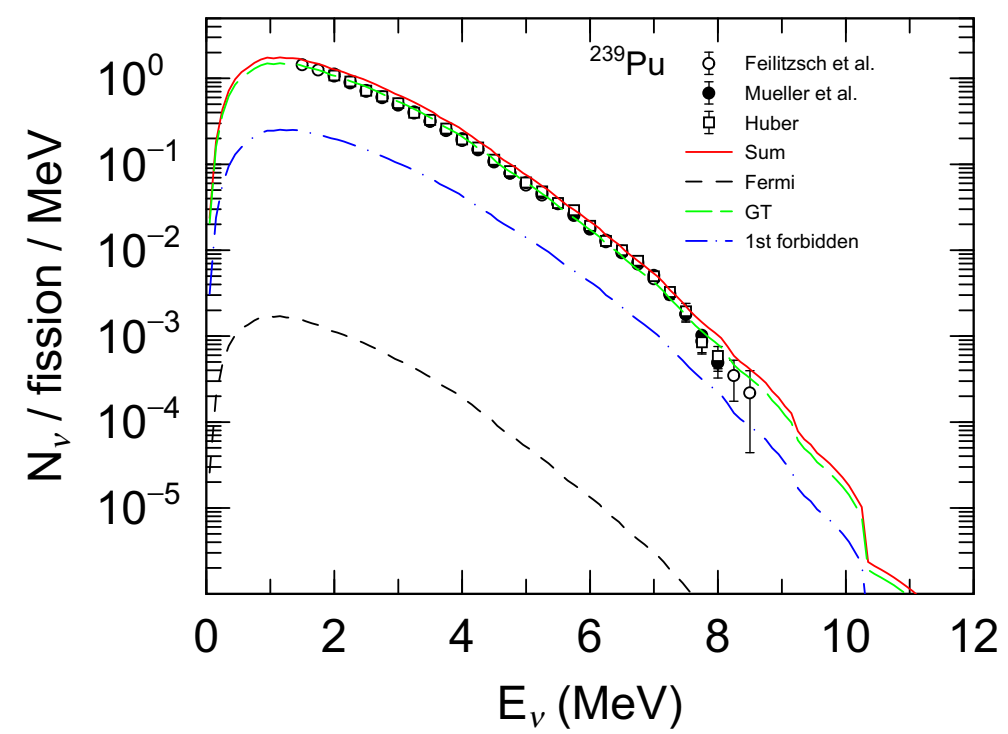

Figure 3. Calculated antineutrino spectrum from ${ }^{239} \mathrm{Pu}$ sample and its component-wise breakdown. For reference are shown three spectra converted from the electron spectrum measured by Feilitzsch et al. 


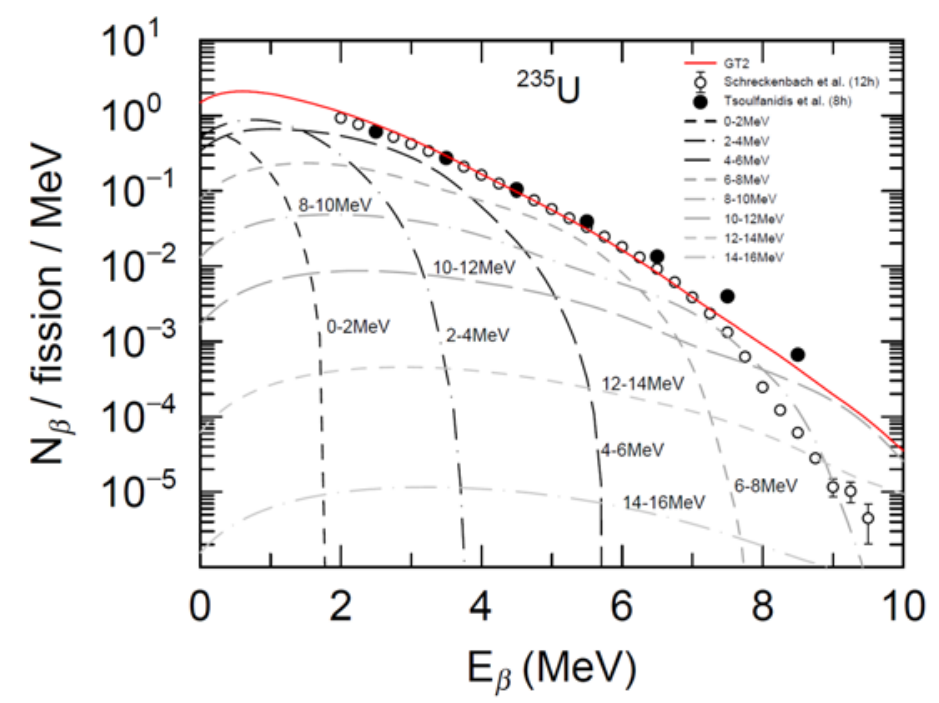

Figure 4. Calculated antineutrino spectrum from ${ }^{235} \mathrm{U}$ sample and its component-wise breakdown. For reference are shown three spectra converted from the electron spectrum measured by Schreckenbach et al.

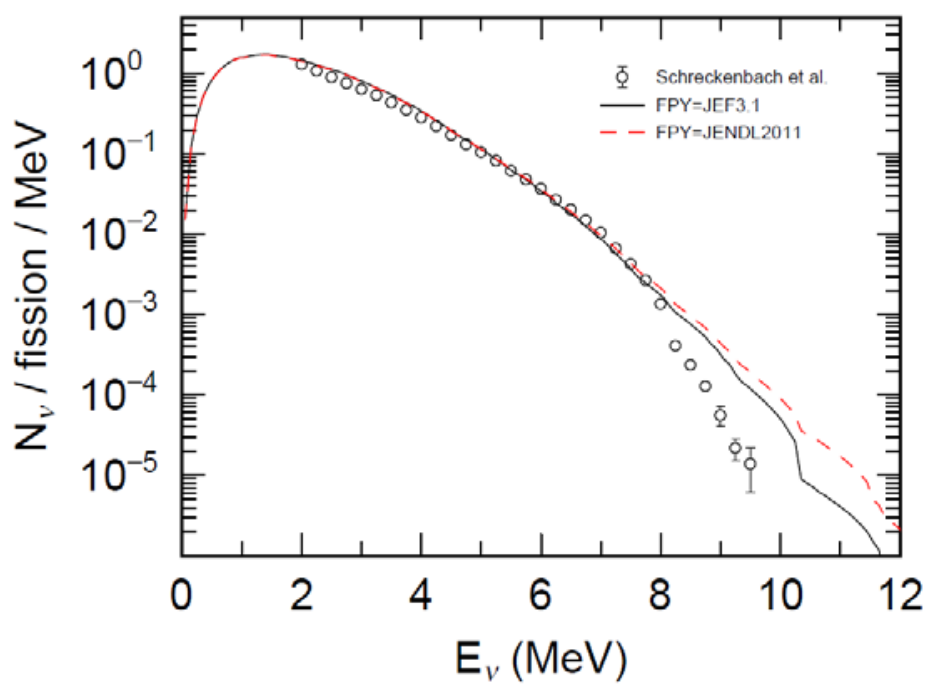

Figure 5. Change induced by replacement of JENDL FP yileds by JEFF yields in calculated antineutrino spectrum from ${ }^{235} \mathrm{U}$ 


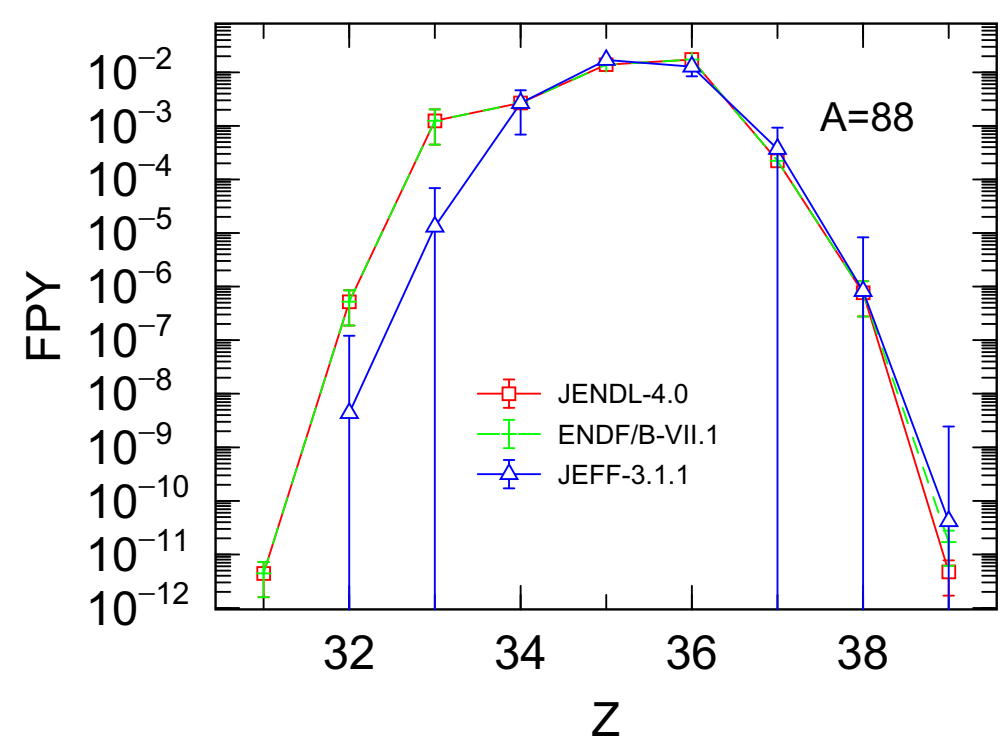

Figure 6. Charge yield for thermal-neutron induced fission of ${ }^{235} \mathrm{U}(A=88)$

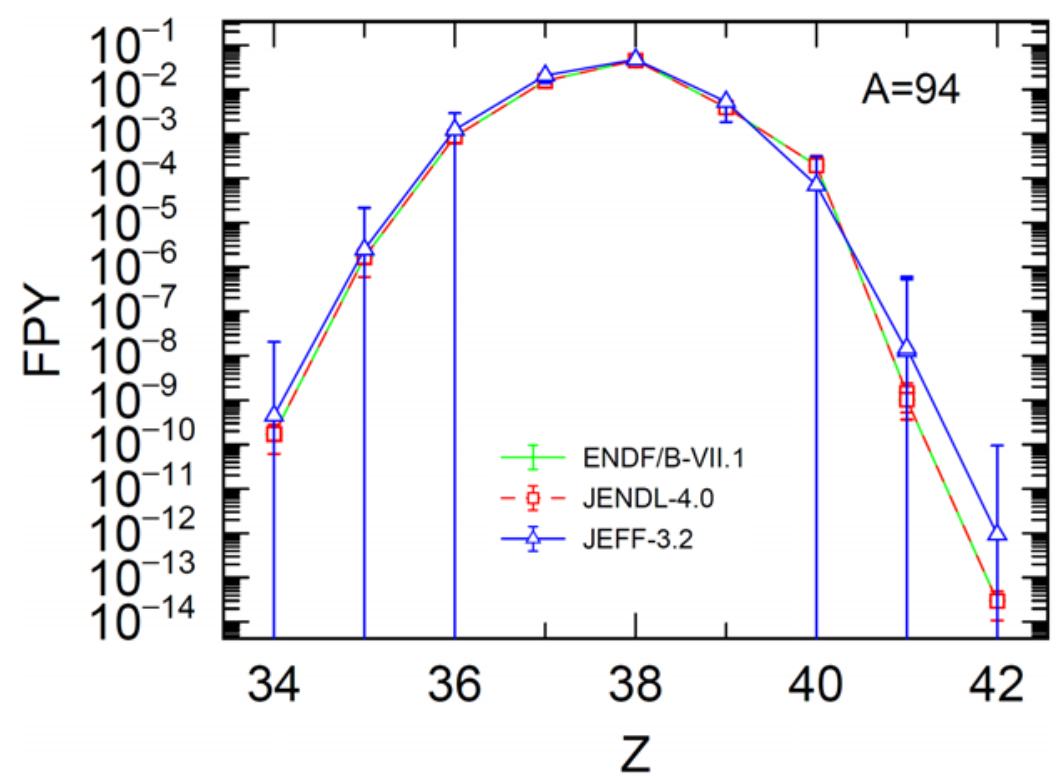

Figure 7. Charge yield for thermal-neutron induced fission of ${ }^{235} \mathrm{U}(A=94)$ 